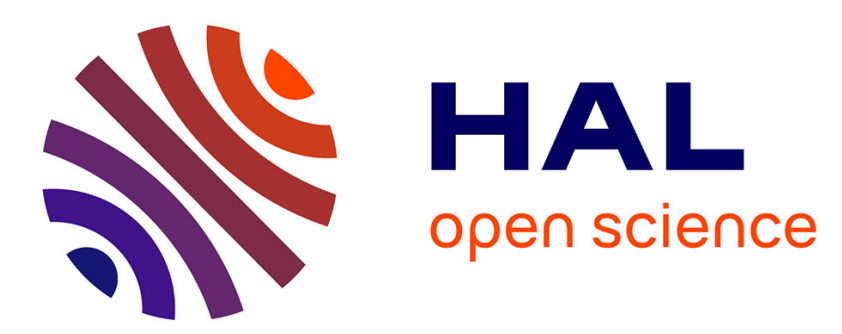

\title{
An LMI solution for a class of robust open-loop problems
}

Benoit Bayon, Gérard Scorletti, Eric Blanco

\section{To cite this version:}

Benoit Bayon, Gérard Scorletti, Eric Blanco. An LMI solution for a class of robust open-loop problems. 2012 IEEE ACC, Jun 2012, Montréal, Canada. pp.5234-5239, 10.1109/ACC.2012.6315092 . hal00793954

\section{HAL Id: hal-00793954 \\ https://hal.science/hal-00793954}

Submitted on 2 Jan 2014

HAL is a multi-disciplinary open access archive for the deposit and dissemination of scientific research documents, whether they are published or not. The documents may come from teaching and research institutions in France or abroad, or from public or private research centers.
L'archive ouverte pluridisciplinaire HAL, est destinée au dépôt et à la diffusion de documents scientifiques de niveau recherche, publiés ou non, émanant des établissements d'enseignement et de recherche français ou étrangers, des laboratoires publics ou privés. 


\title{
An LMI solution for a class of robust open-loop problems
}

\author{
Benoit Bayon, Gérard Scorletti, Eric Blanco ${ }^{1}$
}

\begin{abstract}
The robust filter design and the robust feedforward controller design are particular cases of a larger class of problems: the robust open-loop problems. In this article, we consider a class of uncertain open-loop plants, where a filter needs to be designed to ensure that the plant satisfies chosen specifications. The representation of uncertainties is made in a very general framework: the Linear Fractional Transformation (LFT). Associated with the Dynamic Integral Quadratic Constraints framework, it allows the consideration of many classes of structured uncertainties. This paper proves that the design of a filter ensuring a robust $L_{2}$-gain or $\mathrm{H}_{2}$ performance for the complete plant can be expressed as a convex optimization problem involving Linear Matrix Inequalities Constraints which can be solved using an efficient algorithm.
\end{abstract}

\section{INTRODUCTION}

The class of open-loop plants under consideration is presented in figure 1. In this problem, a filter $F$ (to be synthesized), is placed between two systems $G$ (referred to as the input system) and $H$ (referred to as the output system). This plant is referred to as an open-loop plant as no feedback is acting between the elements $G, F$, and $H$ in the plant. The uncertainty affects only the input system $G$ and is represented in an LFT framework through the uncertainty block $\Delta$.

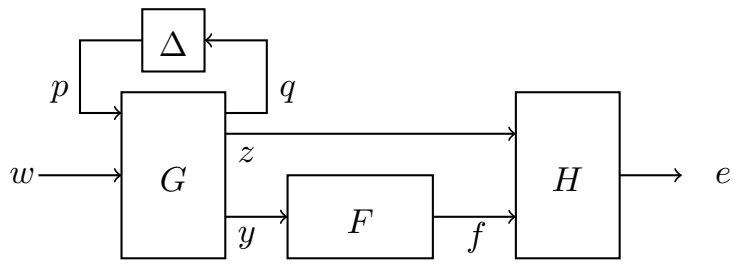

Fig. 1. Robust Open-Loop Problem

In many applications the design of open-loop elements (such as $F$ ) is a critical issue. The synthesis needs to ensure that the complete plant satisfies specifications. This problem recovers the case of the robust filtering [15] and its dual problem, the robust feed-forward case [6]. This also recovers cases in power electronics such as the design of passive elements in an energy transfer line [5]. In these cases the sensitivity of the complete plant to coupling factors or loads is a serious topic.

When models of the plant elements are supposed to be a perfect representation of the reality, i.e no uncertainty affects $G$, the design of a filter is a particular problem of control.

\footnotetext{
${ }^{1}$ The authors are with Laboratoire Ampère, Ecole Centrale de Lyon, Université de Lyon, 36 avenue Guy de Collongue, 69134 Ecully Cedex, France. For correspondance, adress to benoit.bayon@ec-lyon.fr, gerard.scorletti@ec-lyon.fr, eric.blanco@ec-lyon.fr
}

Many solutions have been proposed involving Riccati equation [3] or Linear Matrix Inequalities (LMIs) [4], [13] ensuring that the open-loop satisfies various specifications. But these approaches do not consider the modeling error which potentially cause great performance degradations when the designed controller is implemented on the real system. The gap between the real system and its model can be represented with uncertainties to deal with this major issue. The robust problem is to find a controller (for the closed-loop case) or a filter (for the open-loop case) which ensures a guaranteed performance for all the systems represented by the uncertain model.

Many interesting cases can be modeled using Linear Fractional Transformation (LFT) [18]. In this very general framework, the uncertain model is a rational function of several uncertainties. These systems are represented as an LFT of an uncertain block $\Delta$ by a nominal model. The uncertain block is structured (block-diagonal) allowing to take charge of several uncertainties of different classes at once. In this framework, the uncertain block is related to the nominal system using Integral Quadratic Constraints (IQCs) [8]. These IQCs allow to have an input-output characterization of systems and robust performance analysis tools [14].

While the robust performance analysis is convex, the general robust controller synthesis problem is proved to be non-convex. But for specific open-loop cases, the synthesis problem is convex. In [16] results were proposed for the synthesis of robust filters for Linear-Time-Varying (LTV) parametric and dynamic uncertainties using the LFT/IQC framework with static IQCs. More general results were proposed in [15], for the design of $L_{2}$-gain sub-optimal filters and feed-forward controllers, for parametric and dynamic uncertainties, with the use of Dynamic IQCs. Compared to static IQCs, the use of Dynamic IQCs allows to take into account more types of uncertainties, such as LinearTime-Invariant (LTI) parametric and dynamic uncertainties, non-linearities or delays [8]. More general results were proposed for generalized IQCs, for the design of $\mathrm{H}_{2}$ and $L_{2}$ gain suboptimal filters [14] and feed-forward controllers [7]. A limitation with these results is that they cannot take into account any system at the output of the filter. The system $H$ needs to have the form $[W(j \omega)-W(j \omega)]$, where $W$ is a stable transfer function with a stable inverse, which is not always the case [9]. And even for this case, the order of the designed filter is greater than necessary, except in [17], where weigths can be taken into account without growth of order for the deisgned filter.

When the control channel is not affected by uncertainties 
the controller synthesis problem is proved to be convex [12] using dynamic IQCs. A framework has also been developed to tackle this problem. It allows for example the design of observers for uncertain systems [1]. Unfortunately this general framework also provides filters of order greater than necessary for simpler cases such as the one under consideration in this paper.

To overcome this problem, we present solutions for the class of robust open-loop plants presented in figure 1, which is a particular case of the problem presented in [12], and more general than the problem under consideration in [15], [14]. The representation of uncertainties is made using general dynamic IQCs and allows the consideration of many classes of uncertainties.

We present two theorems allowing to synthesize filters that ensure an upper bound on a $H_{2}$ and $L_{2}$-gain performance on the complete open-loop plant. For the case of the robust weighted filtering problems, the filter designed are smaller in terms of order compared to the one proposed for [15], [14], [12]. These smaller orders are interesting in terms of real-time implementation. It will allow to synthesize simpler robust filters ensuring a performance for variant coupling factors and loads for the design of passive elements in energy transfer lines. Additionally, any matrices of transfer functions can be considered at the end of the plant. Finally, the case where the uncertainty affects the output system $H$ can be tackled using the solutions presented here, as it is the dual problem of the one under consideration. The feed-forward controller designed with these solutions will be of smaller order than the ones designed with the methods presented in [7].

\section{Notations}

$A^{T}$ is the transpose of the matrix $A, A^{*}$ its transpose conjugate. $(\diamond)^{*} X A$ denotes $(A)^{*} X A$. In a matrix $\diamond$ also denotes a symetric element. We also have $A+A^{T}=A+(\bullet)^{T}$

$\Pi(j \omega)$ defines the central term of an Integral Quadratic Constraint, and can be factorized as $[\diamond]^{*}\left[\begin{array}{ll}\Phi_{11} & \Phi_{12} \\ \Phi_{12}^{T} & \Phi_{22}\end{array}\right]\left[\begin{array}{cc}K(j \omega) & 0 \\ 0 & K(j \omega)\end{array}\right] . \quad$ The state space representation of the matrix of transfer function $C(j \omega-A)^{-1} B+D$ is denoted $\left[\begin{array}{c|c}A & B \\ \hline C & D\end{array}\right] \cdot P$ denotes a matrix introduced by the Kalman-Yakubovitch-Popov lemma. Finally $T_{w e}$ denotes the transfer function from $w$ to $e$, and $\left\|T_{w e}\right\|_{2}$ denotes the $H_{2}$ norm of this transfer function, while $\left\|T_{w e}\right\|_{i 2}$ denotes the norm induced by the $L_{2}$ space of signals. The variables of the optimization problems involving LMIs are written in bold.

\section{Problem Definition}

Consider the plant presented in figure 1. $G$ is subject to a non-measured input $w \in \mathbb{R}^{n_{w}}$. This system feeds a filter $F$ (to be synthesized) through the signal $y \in \mathbb{R}^{n_{y}}$ and $H$ through $z \in \mathbb{R}^{n_{z}}$. $H$ is also fed by the output of the filter $f \in \mathbb{R}^{n_{f}}$. The inputs and outputs of the uncertain block $\Delta$ are respectively $q \in \mathbb{R}^{n_{q}}$ and $p \in \mathbb{R}^{n_{p}}$. To consider many classes of uncertainties, we consider that the uncertain block $\Delta$ satisfies the Integral Quadratic Constraint defined by

$$
\int_{-\infty}^{\infty}(\diamond)^{T} \Pi(j \omega)\left[\begin{array}{c}
x(j \omega) \\
\Delta(x)(j \omega)
\end{array}\right] d \omega, \quad \forall x \in L_{2}
$$

If $\Delta$ is a structured uncertainty, then $\Pi(j \omega)$ is also a structured IQC, and its structure depends on the uncertainties under consideration [15]. The transfer function $T_{w e}$ from $w$ to $e$ is under consideration:

$$
T_{w e}=H(j \omega)\left[\begin{array}{cc}
I & 0 \\
0 & F(j \omega)
\end{array}\right](G(j \omega) \star \Delta)
$$

To characterize $T_{w e}$ we consider two norms on systems.

- For LTI systems the $H_{2}$ norm represents the energy of the impulse response. As the IQC presented equation 1 can allows to consider uncertainties such as delays, nonlinearities, we consider a generalization of the $\mathrm{H}_{2}$-norm based on the output signal of the system, considering as input an impulsion [14].

- The $L_{2}$-gain norm is defined as a worst-case performance along the frequency response of a system. In this case, $w$ is a signal of $L_{2}$-gain norm less than one.

The $\mathrm{H}_{2}$ robust open-loop problem is then:

For a given $\gamma>0$, find if a filter $F$ exists (and compute it) so that $\forall \Delta$ which satisfies (1), $\left\|T_{w e}\right\|_{2}<\gamma$.

The $L_{2}$-gain robust open-loop problem is then:

For a given $\gamma>0$, find if a filter $F$ exists (and compute it) so that $\forall \Delta$ which satisfies (1), $\left\|T_{w e}\right\|_{i_{2}}<\gamma$.

\section{A. Sketch of robust performance analysis using IQCs}

The basic results for analysis of uncertain systems using IQCs are presented here. The following fundamental theorem is presented first.

Theorem 2.1: Stability Analysis theorem [8]

Let $G$ be stable, and let $\Delta$ be a bounded causal operator. Assume that

1) for every $\tau \in[0,1]$, the interconnection of $G$ and $\Delta$ is well-posed;

2) for every $\tau \in[0,1]$, the IQC defined by $\Pi$ is satisfied by $\tau \Delta$

3) There exists $\epsilon>0$ such that

$$
\left[\begin{array}{c}
G(j \omega) \\
I
\end{array}\right]^{*} \Pi(j \omega)\left[\begin{array}{c}
G(j \omega) \\
I
\end{array}\right] \leq-\epsilon I, \forall \omega \in \mathbb{R}
$$

Then, the feedback interconnection of $G$ and $\Delta$ is stable.

To test the stability of a given interconnection, one has to find $\Pi(j \omega)$ : this is a feasibility problem. This is hardly feasible as all the matrices of transfer functions of every order are candidates which means the number of variables of the optimization problem is infinite. Moreover, the inequality presented equation (2) has to be tested for all frequencies which means an infinite number of constraints. To handle these issues the common path [15], [14] is as follows: 
- Restrict the matrix of transfer function $\Pi(j \omega)$ to a finite span of matrices of transfer functions, of a fixed order.

- Use the celebrated Kalman-Yakubovitch-Popov (KYP) Lemma [11] to test all the frequencies at once: the constraint is recast as a Linear Matrix Inequality constraint. The optimization problem becomes then a finitedimensional optimization problems, with a finite number of constraints, which can be solved using an efficient algorithm [2].

An example of the application of these two steps is presented here. Consider the equation (2). We restrict $\Pi(j \omega)$ to the matrices of transfer function such as

$$
[\diamond]^{*}\left[\begin{array}{ll}
\Phi_{11} & \Phi_{12} \\
\Phi_{12}^{T} & \Phi_{22}
\end{array}\right]\left[\begin{array}{cc}
K(j \omega) & 0 \\
0 & K(j \omega)
\end{array}\right]
$$

To generate all the candidates, the choice of $K(j \omega)$ is highly non-unique. For example $K(j \omega)=$ $\left.\left[\begin{array}{llll}j \omega^{n} & \ldots & j \omega & 1\end{array}\right]^{T} \otimes I_{n_{q}}\right) / d(j \omega)$ is a suitable basis, where $d(j \omega)$ is a fixed Hurwitz polynomial with $n$ poles. With this representation, the order of $\Pi(j w)$ is restricted to $2 n$. This factorization introduces some conservatism, but this conservatism decreases dramatically when the order chosen for the IQC increases [14]. For specific structures as parametric and dynamic LTI/LTV structured uncertainties one can refer to [15] for economical parametrization to reduce the computation time.

$$
[\diamond]^{*}\left[\begin{array}{ll}
\Phi_{11} & \Phi_{12} \\
\Phi_{12}^{T} & \Phi_{22}
\end{array}\right]\left[\begin{array}{c}
K(j \omega) G(j \omega) \\
K(j \omega)
\end{array}\right] \leq-\epsilon I, \forall \omega \in \mathbb{R}
$$

Applying the KYP Lemma [11], the constraint (4) holds if $\mathbf{P}=\mathbf{P}^{T}, \boldsymbol{\Phi}$ exist so that the condition (5) holds.

$$
[\diamond]^{T}\left[\begin{array}{cc|cc}
0 & \mathbf{P} & 0 & 0 \\
\mathbf{P} & 0 & 0 & 0 \\
\hline 0 & 0 & \mathbf{\Phi}_{\mathbf{1 1}} & \mathbf{\Phi}_{\mathbf{1 2}} \\
0 & 0 & \mathbf{\Phi}_{\mathbf{1 2}}{ }^{T} & \mathbf{\Phi}_{\mathbf{2 2}}
\end{array}\right]\left[\begin{array}{cc}
I & 0 \\
\mathcal{A} & \mathcal{B} \\
\hline \mathcal{C} & \mathcal{D}
\end{array}\right]<0
$$

$$
\left[\begin{array}{l|l}
\mathcal{A} & \mathcal{B} \\
\hline \mathcal{C} & \mathcal{D}
\end{array}\right] \text { is a state space form of }\left[\begin{array}{c}
K(j \omega) G(j \omega) \\
K(j \omega)
\end{array}\right] \text {. }
$$

- $G(j \omega)=C(j \omega I-A)^{-1} B+D$

- $K(j \omega)=C_{K}\left(j \omega I-A_{K}\right)^{-1} B_{K}+D_{K}$.

$$
\left[\begin{array}{c|c}
\mathcal{A} & \mathcal{B} \\
\hline \mathcal{C} & \mathcal{D}
\end{array}\right]=\left[\begin{array}{ccc|c}
A_{K} & 0 & 0 & B_{K} \\
0 & A_{K} & B_{K} C & B_{K} D \\
0 & 0 & A & B \\
\hline 0 & C_{K} & D_{K} C & D_{K} D \\
C_{K} & 0 & 0 & D_{K}
\end{array}\right]
$$

From equation (1), we can assume that $K^{*}(j \omega) \Phi_{11} K(j \omega)>0$, and this property has to be ensured with the factorization presented equation (3). Applying the KYP Lemma [11], this constraint holds if $\mathbf{P}_{\mathbf{K}}=\mathbf{P}_{\mathbf{K}}{ }^{T}$ exists so that the condition 5 holds.

$$
[\diamond]^{T}\left[\begin{array}{cc|c}
0 & \mathbf{P}_{\mathbf{K}} & 0 \\
\mathbf{P}_{\mathbf{K}} & 0 & 0 \\
\hline 0 & 0 & \mathbf{\Phi}_{\mathbf{1 1}}
\end{array}\right]\left[\begin{array}{cc}
I & 0 \\
A_{K} & B_{K} \\
\hline C_{K} & D_{K}
\end{array}\right]>0
$$

These operations allow to transform an infinite dimensional optimization problem into a finite dimensional convex optimization problem with a finite number of constraints. The initial condition (2) holds if the constraints (5),(6) holds. Note that these conditions are only sufficient because the factorization and the restriction of order induce some conservatism. But IQCs of small orders have proven to be efficient enough to reduce drastically the conservatism [14].

\section{B. Robust Performance analysis theorems}

These results lead to interesting results in robust performance analysis. We consider the system presented figure 2 .

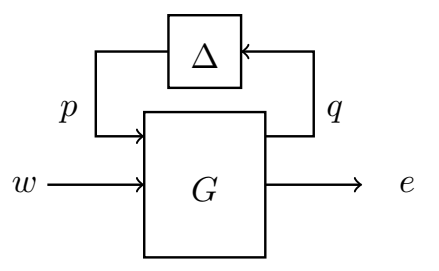

Fig. 2. Uncertain system

$G=\left[\begin{array}{l}C_{q} \\ C_{e}\end{array}\right](j \omega I-A)^{-1}\left[\begin{array}{ll}B_{p} & B_{w}\end{array}\right]+\left[\begin{array}{cc}D_{q p} & D_{q w} \\ D_{e p} & D_{e w}\end{array}\right]$

The condition (1) holds for $\Delta$. The objective is to have conditions to test a worst-case performance on the transfer function $T_{e w}$. The framework presented in the previous subsection has led to useful results presented in theorem 2.2 and 2.3.

Theorem 2.2: Robust $L_{2}$-gain Performance [14] Let $G$ be stable, the tranfer function $T_{w e}$ has an $L_{2}$-gain less than $\gamma$ if $\mathbf{P}=\mathbf{P}^{T}, \mathbf{\Phi}=\left[\begin{array}{cc}\boldsymbol{\Phi}_{11} & \boldsymbol{\Phi}_{12} \\ \boldsymbol{\Phi}_{12}{ }^{T} & \boldsymbol{\Phi}_{\mathbf{2 2}}\end{array}\right]$ exist so that the conditions $(6,7)$ hold.

$$
[\diamond]^{T}\left[\begin{array}{ccccc}
0 & \mathbf{P} & 0 & 0 & 0 \\
\mathbf{P} & 0 & 0 & 0 & 0 \\
0 & 0 & \mathbf{\Phi} & 0 & 0 \\
0 & 0 & 0 & I & 0 \\
0 & 0 & 0 & 0 & -\gamma^{2} I
\end{array}\right]\left[\begin{array}{cc}
I & 0 \\
\mathcal{A} & \mathcal{B} \\
\mathcal{C}_{q} & \mathcal{D}_{q} \\
\mathcal{C}_{e} & \mathcal{D}_{e} \\
0 & \mathcal{D}_{w}
\end{array}\right]<0
$$

with the following matrices:

$$
\left[\begin{array}{c|c}
\mathcal{A} & \mathcal{B} \\
\hline \mathcal{C}_{q} & \mathcal{D}_{q} \\
\hline \mathcal{C}_{e} & \mathcal{D}_{e} \\
\hline 0 & \mathcal{D}_{w}
\end{array}\right]=\left[\begin{array}{ccc|cc}
A_{K} & 0 & 0 & B_{K} & 0 \\
0 & A_{K} & B_{K} C_{q} & B_{K} D_{q p} & B_{K} D_{q w} \\
0 & 0 & A & B_{p} & B_{w} \\
\hline 0 & C_{K} & D_{K} C_{q} & D_{K} D_{q p} & D_{K} D_{q w} \\
C_{K} & 0 & 0 & D_{K} & 0 \\
\hline 0 & 0 & C_{e} & D_{e p} & D_{e w} \\
\hline 0 & 0 & 0 & 0 & I
\end{array}\right]
$$

Theorem 2.3: Robust $H_{2}$ performance [14]

Let $G$ be stable, the tranfer function $T_{w e}$ has an $H_{2}$ norm less than $\gamma$ if $\mathbf{P}=\mathbf{P}^{T}, \boldsymbol{\Phi}=\left[\begin{array}{cc}\boldsymbol{\Phi}_{11} & \boldsymbol{\Phi}_{12} \\ \boldsymbol{\Phi}_{12} & \boldsymbol{\Phi}_{\mathbf{2 2}}\end{array}\right], \mathbf{Q}=\mathbf{Q}^{T}$ exist so that the conditions $(6,8-12)$ hold.

$$
\begin{gathered}
{[\diamond]^{T}\left[\begin{array}{cccc}
0 & \mathbf{P} & 0 & 0 \\
\mathbf{P} & 0 & 0 & 0 \\
0 & 0 & \mathbf{\Phi} & 0 \\
0 & 0 & 0 & I
\end{array}\right]\left[\begin{array}{cc}
I & 0 \\
\mathcal{A} & \mathcal{B}_{p} \\
\mathcal{C}_{q} & \mathcal{D}_{q p} \\
\mathcal{C}_{e} & \mathcal{D}_{e p}
\end{array}\right]<0} \\
\left(\begin{array}{cc}
\mathbf{P} & \mathbf{P} \mathcal{B}_{w} \\
\mathcal{B}_{w}^{T} \mathbf{P} & \mathbf{Q}
\end{array}\right)>0
\end{gathered}
$$




$$
\begin{gathered}
\operatorname{Trace}(Q)<\gamma^{2} \\
D_{q w}=0 \\
D_{e w}=0
\end{gathered}
$$

with the following matrices:

$\left[\begin{array}{c|c|c}\mathcal{A} & \mathcal{B}_{p} & \mathcal{B}_{w} \\ \hline \mathcal{C}_{q} & \mathcal{D}_{q p} & 0 \\ \hline \mathcal{C}_{e} & \mathcal{D}_{e p} & 0\end{array}\right]=\left[\begin{array}{ccc|c|c}A_{K} & 0 & 0 & B_{K} & 0 \\ 0 & A_{K} & B_{K} C_{q} & B_{K} D_{q p} & 0 \\ 0 & 0 & A & B_{p} & B_{w} \\ \hline 0 & C_{K} & D_{K} C_{q} & D_{K} D_{q p} & 0 \\ C_{K} & 0 & 0 & D_{K} & 0 \\ \hline 0 & 0 & C_{e} & D_{e p} & 0\end{array}\right]$

For a given system and a given $\gamma$, constraints given in theorems 2.2 and 2.3 define feasibility problems involving LMIs constraints. This can also be recast as a minimization problem of a cost function minimizing $x=\gamma^{2}$, to find the lower upper-bound reachable in this framework on the worstcase performance.

\section{MAIn RESUlts}

In this section we consider the open-loop plant presented in figure 1. Two theorems are revealed, allowing to test the existence of a solution for the robust open-loop problem for a given level of $H_{2}$ or $L_{2}$-gain performance. The corresponding filter can be computed from the solution of the optimization problems. We have the following definitions:

$G(j \omega)=\left[\begin{array}{l}C_{q} \\ C_{z} \\ C_{y}\end{array}\right](j \omega I-A)^{-1}\left[\begin{array}{ll}B_{p} & B_{w}\end{array}\right]+\left[\begin{array}{cc}D_{q p} & D_{q w} \\ D_{z p} & D_{z w} \\ D_{y p} & D_{y w}\end{array}\right]$ $H(j \omega)=\left[C_{e}\right]\left(j \omega I-A_{H}\right)^{-1}\left[\begin{array}{ll}B_{z} & B_{f}\end{array}\right]+\left[\begin{array}{ll}D_{e z} & D_{e f}\end{array}\right]$

Theorem 3.1: Robust $L_{2}$-gain Open-Loop Synthesis

For a given $\gamma>0$, if (1) holds $\forall \Delta$, a filter exists so that $\left\|T_{w e}\right\|_{i 2}<\gamma$, if $\mathbf{Z}_{\mathbf{1}}=\mathbf{Z}_{\mathbf{1}}^{\mathbf{T}}, \mathbf{Z}_{\mathbf{2}}, \mathbf{Z}_{\mathbf{3}}=\mathbf{Z}_{\mathbf{3}}^{\mathbf{T}}, \mathbf{P}_{\mathbf{1}}=$ $\left[\begin{array}{ll}\mathbf{P}_{11} & \mathbf{P}_{12} \\ \mathbf{P}_{12}^{\mathbf{T}} & \mathbf{P}_{22}\end{array}\right], \mathbf{F}, \mathbf{P}_{\mathbf{K}}=\mathbf{P}_{\mathbf{K}}^{\mathbf{T}}, \boldsymbol{\Phi}=\left[\begin{array}{ll}\boldsymbol{\Phi}_{11} & \boldsymbol{\Phi}_{12} \\ \boldsymbol{\Phi}_{12}^{\mathrm{T}} & \boldsymbol{\Phi}_{22}\end{array}\right]$ of appropriates dimensions exist so that conditions (13), (14), (15) hold.

$$
\mathcal{L}_{1}+\mathcal{L}_{1}^{T}+\mathcal{L}_{2}+\mathcal{L}_{2}^{T}+\mathcal{L}_{3}+\mathcal{L}_{3}^{T}+\mathcal{L}_{\Phi}<0
$$

with the following matrices:

$$
\begin{aligned}
\mathcal{L}_{1} & =\left[\begin{array}{ccc}
\mathbf{Z}_{1} & 0 & 0 \\
-\mathbf{Z}_{2}^{\mathbf{T}} & I & 0 \\
\mathbf{P}_{11} & \mathbf{P}_{12} & 0 \\
\mathbf{P}_{12}^{\mathbf{T}} & \mathbf{P}_{\mathbf{2 2}} & 0 \\
0 & 0 & 0 \\
0 & 0 & I
\end{array}\right]\left[\begin{array}{ccc}
A_{G} & 0 & B_{G} \\
B_{z} C_{G 2} & A_{H} & B_{z} D_{G 2} \\
D_{e z} C_{G 2} & C_{e} & D_{e z} D_{G 2}
\end{array}\right] U_{L 1} \\
U_{L 1} & =\left[\begin{array}{llllll}
I & 0 & I & 0 & 0 & 0 \\
0 & 0 & 0 & I & 0 & 0 \\
0 & 0 & 0 & 0 & I & 0
\end{array}\right]
\end{aligned}
$$$$
\mathcal{L}_{2=}\left[\begin{array}{ll}
0 & 0 \\
I & 0 \\
0 & 0 \\
0 & 0 \\
0 & 0 \\
0 & I
\end{array}\right]\left[\begin{array}{c}
A_{H} \\
C_{e}
\end{array}\right]\left[\begin{array}{llllll}
\mathbf{Z}_{2}^{\mathbf{T}} & \mathbf{Z}_{3} & 0 & 0 & 0 & 0
\end{array}\right]
$$$$
\mathcal{L}_{3}=\left[\begin{array}{ccc}
0 & 0 & 0 \\
0 & 0 & B_{f} \\
I & 0 & 0 \\
0 & I & 0 \\
0 & 0 & 0 \\
0 & 0 & D_{e f}
\end{array}\right] \mathbf{F}\left[\begin{array}{ccc}
I & 0 & 0 \\
0 & I & 0 \\
0 & 0 & C_{G 3}^{T} \\
0 & 0 & 0 \\
0 & 0 & D_{G 3}^{T} \\
0 & 0 & 0
\end{array}\right]^{T}
$$

$$
\begin{gathered}
\mathcal{L}_{\Phi}=[\diamond]^{T}\left[\begin{array}{ccc}
\mathbf{\Phi} & 0 & 0 \\
0 & -\gamma^{2} I & 0 \\
0 & 0 & -I
\end{array}\right]\left[\begin{array}{cccccc}
C_{\phi} & 0 & C_{\phi} & 0 & D_{\phi} & 0 \\
0 & 0 & 0 & 0 & D_{w} & 0 \\
0 & 0 & 0 & 0 & 0 & I
\end{array}\right] \\
{[\diamond]^{T}\left[\begin{array}{cc|c}
0 & \mathbf{P}_{\mathbf{K}} & 0 \\
\mathbf{P}_{\mathbf{K}} & 0 & 0 \\
\hline 0 & 0 & \mathbf{\Phi}_{\mathbf{1 1}}
\end{array}\right]\left[\begin{array}{ccc}
I & 0 \\
A_{K} & B_{K} \\
\hline C_{K} & D_{K}
\end{array}\right]<0} \\
{\left[\begin{array}{cccc}
\mathbf{Z}_{\mathbf{1}} & 0 & \mathbf{Z}_{\mathbf{1}} & 0 \\
\diamond & \mathbf{Z}_{\mathbf{3}} & -\mathbf{Z}_{\mathbf{2}}^{\mathbf{T}} & I \\
\diamond & \diamond & \mathbf{P}_{\mathbf{1 1}} & \mathbf{P}_{\mathbf{1 2}} \\
\diamond & \diamond & \diamond & \mathbf{P}_{\mathbf{2 2}}
\end{array}\right]>0}
\end{gathered}
$$

with the following matrices:

$$
\left[\begin{array}{c|c}
A_{G} & B_{G} \\
\hline C_{\Phi} & D_{\Phi} \\
\hline C_{G 2} & D_{G 2} \\
\hline C_{G 3} & D_{G 3} \\
\hline 0 & D_{w}
\end{array}\right]=\left[\begin{array}{ccc|cc}
A_{K} & 0 & 0 & B_{K} & 0 \\
0 & A_{K} & B_{K} C & B_{K} D_{q p} & B_{K} D_{q w} \\
0 & 0 & A & B_{p} & B_{w} \\
\hline 0 & C_{K} & D_{K} C_{q} & D_{K} D_{q p} & D_{K} D_{q w} \\
C_{K} & 0 & 0 & D_{K} & 0 \\
\hline 0 & 0 & C_{z} & D_{z p} & D_{z w} \\
\hline 0 & 0 & C_{y} & D_{y p} & D_{y w} \\
\hline 0 & 0 & 0 & 0 & I
\end{array}\right]
$$

Proof: Theorem 2.2 is applied on the system presented in figure 1 . The first condition is:

$$
[\diamond]^{T}\left[\begin{array}{ccccc}
0 & P & 0 & 0 & 0 \\
P & 0 & 0 & 0 & 0 \\
0 & 0 & \boldsymbol{\Phi} & 0 & 0 \\
0 & 0 & 0 & I & 0 \\
0 & 0 & 0 & 0 & -\gamma^{2} I
\end{array}\right]\left[\begin{array}{cc}
I & 0 \\
\mathcal{A} & \mathcal{B} \\
\mathcal{C}_{q} & \mathcal{D}_{q} \\
\mathcal{C}_{e} & \mathcal{D}_{e} \\
0 & \mathcal{D}_{w}
\end{array}\right]<0
$$

with

$$
\begin{aligned}
& {\left[\begin{array}{c}
\mathcal{A} \\
\hline \frac{\mathcal{C}_{q}}{\mathcal{C}_{e}}
\end{array}\right]=\left[\begin{array}{ccc}
A_{G} & 0 & 0 \\
B_{z} C_{G 2}+B_{f} D_{F} C_{G 3} & A_{H} & B_{z} C_{F} \\
B_{F} C_{G 3} & 0 & A_{F} \\
\hline C_{\Phi} & 0 & 0 \\
\hline D_{e z} C_{G 2}+D_{e f} D_{F} C_{G 3} & C_{e} & D_{e f} C_{F}
\end{array}\right]} \\
& {\left[\begin{array}{c}
B_{G} \\
\hline \mathcal{D}_{q} \\
\hline \mathcal{D}_{e} \\
\hline \mathcal{D}_{w}
\end{array}\right]=\left[\begin{array}{c}
B_{z} D_{G 2}+B_{f} D_{F} D_{G 3} \\
B_{F} D_{G 3} \\
D_{\Phi} \\
\left.\frac{D_{e z} D_{G 2}+D_{e f} D_{F} D_{G 3}}{0} I\right]
\end{array}\right]}
\end{aligned}
$$

Using a Schur lemma [2, page 28], this can be recast as:

$$
\begin{aligned}
& \overbrace{\left[\begin{array}{ll}
P & 0 \\
0 & 0 \\
0 & I
\end{array}\right]\left[\begin{array}{ccc}
\mathcal{A} & \mathcal{B} & 0 \\
\mathcal{C}_{e} & \mathcal{D}_{e} & 0
\end{array}\right]}^{\Psi_{1}}+\overbrace{(\bullet)^{T}}^{\Psi_{1}^{T}}+\ldots \\
& \overbrace{\ldots(\diamond)^{T}\left[\begin{array}{ccc}
\Phi & 0 & 0 \\
0 & -\gamma^{2} I & 0 \\
0 & 0 & -I
\end{array}\right]\left[\begin{array}{ccc}
\mathcal{C}_{q} & \mathcal{D}_{q} & 0 \\
0 & \mathcal{D}_{w} & 0 \\
0 & 0 & I
\end{array}\right]}^{\Psi_{2}}<0
\end{aligned}
$$

We introduce the partitions of $P$ and its inverse.

$$
\begin{aligned}
& P=\left[\begin{array}{c|c}
\mathbf{P}_{\mathbf{1}} & P_{2} \\
\hline \diamond & P_{33}
\end{array}\right]=\left[\begin{array}{cc|c}
\mathbf{P}_{\mathbf{1 1}} & \mathbf{P}_{\mathbf{1 2}} & P_{13} \\
\diamond & \mathbf{P}_{\mathbf{2 2}} & P_{23} \\
\hline \diamond & \diamond & P_{33}
\end{array}\right] \text { so that } \\
& P \mathcal{A}=\left[\begin{array}{c|c|c}
\mathbf{P}_{\mathbf{1 1}} & \mathbf{P}_{\mathbf{1 2}} & P_{13} \\
\hline \diamond & \mathbf{P}_{\mathbf{2 2}} & P_{23} \\
\hline \diamond & \diamond & P_{33}
\end{array}\right]\left[\begin{array}{c|c|c}
A_{G} & \ldots & \ldots \\
\hline \ldots & A_{H} & \ldots \\
\hline \ldots & \ldots & A_{F}
\end{array}\right] \\
& P^{-1}=Q=\left[\begin{array}{c|c}
\mathbf{Q}_{\mathbf{1}} & Q_{2} \\
\hline \diamond & Q_{33}
\end{array}\right]=\left[\begin{array}{cc|c}
Q_{11} & Q_{12} & Q_{13} \\
\diamond & Q_{22} & Q_{23} \\
\hline \diamond & \diamond & Q_{33}
\end{array}\right]
\end{aligned}
$$


Notice that $P_{33}, Q_{33}, P_{2}, Q_{2}, P_{1}, Q_{1}$ are square matrix of the same dimensions, for the problem to be convex.

A congruent multiplication is made on equation (17) with $\operatorname{diag}(V J, I, I), V$ and $J$ defined equation (18).

$$
J=\left[\begin{array}{ccc}
Q_{11} & Q_{12} & Q_{13} \\
Q_{12}^{T} & Q_{22} & Q_{23} \\
I & 0 & 0 \\
0 & I & 0
\end{array}\right] \quad V=\left[\begin{array}{cccc}
\mathbf{Z}_{\mathbf{1}} & 0 & 0 & 0 \\
-\mathbf{Z}_{2}^{\mathrm{T}} & I & 0 & 0 \\
0 & 0 & I & 0 \\
0 & 0 & 0 & I
\end{array}\right]
$$

$\mathbf{Z}_{\mathbf{1}}=Q_{11}^{-1}, \mathbf{Z}_{\mathbf{2}}=Q_{11}^{-1} Q_{12}, \mathbf{Z}_{\mathbf{3}}=Q_{22}-Q_{12}^{T} Q_{11}^{-1} Q_{12}$.

$V J=\left[\begin{array}{ccc}I & \mathbf{Z}_{2} & R_{1} \\ 0 & \mathbf{Z}_{3} & R_{2} \\ I & 0 & 0 \\ 0 & I & 0\end{array}\right], V J P=\left[\begin{array}{ccc}\mathbf{Z}_{1} & 0 & 0 \\ -\mathbf{Z}_{2}^{\mathbf{T}} & I & 0 \\ \mathbf{P}_{11} & \mathbf{P}_{12} & P_{13} \\ \mathbf{P}_{12}^{\mathbf{T}} & \mathbf{P}_{22} & P_{23}\end{array}\right]$.

Note that this congruent multiplication on the third term $\Psi_{2}$ of equation (17) gives the term $\mathcal{L}_{\Phi}$ of equation (13). The result of this congruent multiplication on the first term $\Psi_{1}$ (and its transpose) is as follows:

$\mathcal{L}_{1}+\mathcal{L}_{2}+\left[\begin{array}{cc}0 & 0 \\ 0 & 0 \\ P_{12} & 0 \\ P_{22} & 0 \\ 0 & 0 \\ 0 & 0\end{array}\right] A_{H}\left[\begin{array}{llllll}\mathbf{Z}_{2}^{\mathbf{T}} & \mathbf{Z}_{3} & 0 & 0 & 0 & 0\end{array}\right] \ldots$
$+\left[\begin{array}{ccc}0 & 0 & 0 \\ I & 0 & 0 \\ P_{12} & P_{13} & 0 \\ P_{22} & P_{23} & 0 \\ 0 & 0 & 0 \\ 0 & 0 & I\end{array}\right]\left[\begin{array}{cc}B_{f} D_{F} & B_{f} C_{F} \\ B_{F} & A_{F} \\ D_{e f} D_{F} & D_{e f} C_{F}\end{array}\right]\left[\begin{array}{cc}C_{G 3}^{T} & R_{1} \\ 0 & R_{2} \\ C_{G 3}^{T} & 0 \\ 0 & 0 \\ D_{G 3} & 0 \\ 0 & 0\end{array}\right]$

Rewrite both last terms as:

$U_{1}\left[\begin{array}{c}{\left[\begin{array}{cc}P_{13} & P_{12} B_{f} \\ P_{23} & P_{22} B_{f} \\ 0 & I\end{array}\right]\left[\begin{array}{ll}A_{F} & B_{F} \\ C_{F} & D_{F}\end{array}\right]\left[\begin{array}{ccc}R_{1}^{T} & R_{2}^{T} & 0 \\ C_{G 3} & 0 & I\end{array}\right]} \\ \cdots+\left[\begin{array}{c}P_{12} \\ P_{22} \\ 0\end{array}\right] A_{H}\left[\begin{array}{lll}\mathbf{Z}_{2}^{\mathrm{T}} & \mathbf{Z}_{3}^{\mathrm{T}} & 0\end{array}\right]\end{array}\right] U_{2}$

with $U_{1}=\left[\begin{array}{ccc}0 & 0 & 0 \\ 0 & 0 & B_{f} \\ I & 0 & 0 \\ 0 & I & 0 \\ 0 & 0 & 0 \\ 0 & 0 & D_{e f}\end{array}\right], U_{2}=\left[\begin{array}{ccc}I & 0 & 0 \\ 0 & I & 0 \\ 0 & 0 & C_{G 3}^{T} \\ 0 & 0 & D_{G 3}^{T} \\ 0 & 0 & 0 \\ 0 & 0 & 0\end{array}\right]^{T}$

Apply the following bijective variable change to get $\mathcal{L}_{3}$.

$\mathbf{F}=\left[\begin{array}{c}{\left[\begin{array}{cc}P_{13} & P_{12} B_{f} \\ P_{23} & P_{22} B_{f} \\ 0 & I\end{array}\right]\left[\begin{array}{ll}A_{F} & B_{F} \\ C_{F} & D_{F}\end{array}\right]\left[\begin{array}{ccc}R_{1}^{T} & R_{2}^{T} & 0 \\ C_{G 3} & 0 & I\end{array}\right]} \\ \cdots+\left[\begin{array}{c}P_{12} \\ P_{22} \\ 0\end{array}\right] A_{H}\left[\begin{array}{lll}\mathbf{Z}_{2}^{\mathrm{T}} & \mathbf{Z}_{3}^{\mathrm{T}} & 0\end{array}\right]\end{array}\right.$

$\mathbf{F}$ has the same size as the original matrices of the state space representation of the filter $\left[\begin{array}{cc}A_{F} & B_{F} \\ C_{F} & D_{F}\end{array}\right]$. To build the filter form the solution of the optimization problem, one has to apply the inverse variable change.

With this congruent multiplication, the condition (13) of the theorem is obtained. The condition (14) is obtained using the theorem (2.2). Finally, the conditions $V J P J^{T} V^{T}$, equation (15) ensure the stability of the filter.
Theorem 3.2: Robust $H_{2}$ Open-Loop Synthesis

For a given $\gamma>0$, if (1) holds $\forall \Delta$, a filter exists so that $\left\|T_{w e}\right\|_{2}<\gamma$, if $\mathbf{Z}_{1}=\mathbf{Z}_{1}^{\mathbf{T}}, \mathbf{Z}_{\mathbf{2}}, \mathbf{Z}_{3}=\mathbf{Z}_{3}^{\mathbf{T}}, \mathbf{P}_{\mathbf{1}}=$ $\left[\begin{array}{ll}\mathbf{P}_{11} & \mathbf{P}_{12} \\ \mathbf{P}_{12}^{\mathrm{T}} & \mathbf{P}_{22}\end{array}\right], \quad \mathbf{F}, \mathbf{P}_{\mathrm{K}}=\mathbf{P}_{\mathrm{K}}^{\mathrm{T}}, \boldsymbol{\Phi}=\left[\begin{array}{ll}\boldsymbol{\Phi}_{11} & \boldsymbol{\Phi}_{12} \\ \boldsymbol{\Phi}_{12}^{\mathrm{T}} & \boldsymbol{\Phi}_{22}\end{array}\right]$, $\mathbf{W}=\mathbf{W}^{\mathbf{T}}$ of appropriates dimensions exist so that conditions (19-24) hold.

$$
\mathcal{L}_{1}+\mathcal{L}_{1}^{T}+\mathcal{L}_{2}+\mathcal{L}_{2}^{T}+\mathcal{L}_{3}+\mathcal{L}_{3}^{T}+\mathcal{L}_{\Phi}<0
$$

$\mathcal{L}_{1}=\left[\begin{array}{ccc}\mathbf{Z}_{1} & 0 & 0 \\ -\mathbf{Z}_{2}^{\mathrm{T}} & I & 0 \\ \mathbf{P}_{11} & \mathbf{P}_{12} & 0 \\ \mathbf{P}_{12}^{\mathrm{T}} & \mathbf{P}_{\mathbf{2 2}} & 0 \\ 0 & 0 & 0 \\ 0 & 0 & I\end{array}\right]\left[\begin{array}{ccc}A_{G} & 0 & B_{G 2 p} \\ B_{z} C_{G 2} & A_{H} & B_{z} D_{G 2 p} \\ D_{e z} C_{G 2} & C_{e} & D_{e z} D_{G 2 p}\end{array}\right] U_{L 1}$ $U_{L 1}=\left[\begin{array}{cccccc}I & 0 & I & 0 & 0 & 0 \\ 0 & 0 & 0 & I & 0 & 0 \\ 0 & 0 & 0 & 0 & I & 0\end{array}\right]$

$\mathcal{L}_{2}=\left[\begin{array}{cc}0 & 0 \\ I & 0 \\ 0 & 0 \\ 0 & 0 \\ 0 & 0 \\ 0 & I\end{array}\right]\left[\begin{array}{c}A_{H} \\ C_{e}\end{array}\right]\left[\begin{array}{llllll}\mathbf{Z}_{2}^{\mathrm{T}} & \mathbf{Z}_{3} & 0 & 0 & 0 & 0\end{array}\right]$ $\mathcal{L}_{3}=\left[\begin{array}{ccc}0 & 0 & 0 \\ 0 & 0 & B_{f} \\ I & 0 & 0 \\ 0 & I & 0 \\ 0 & 0 & 0 \\ 0 & 0 & D_{e f}\end{array}\right] \mathbf{F}\left[\begin{array}{ccc}I & 0 & 0 \\ 0 & I & 0 \\ 0 & 0 & C_{G 3}^{T} \\ 0 & 0 & 0 \\ 0 & 0 & D_{G 3 p}^{T} \\ 0 & 0 & 0\end{array}\right]^{T}$ $\mathcal{L}_{\Phi}=[\diamond]^{T}\left[\begin{array}{cc}\boldsymbol{\Phi} & 0 \\ 0 & -I\end{array}\right]\left[\begin{array}{cccccc}C_{\phi} & 0 & C_{\phi} & 0 & D_{\phi} & 0 \\ 0 & 0 & 0 & 0 & 0 & -I\end{array}\right]$

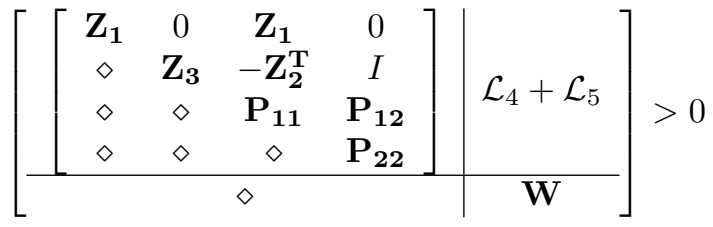

$$
\begin{aligned}
& \mathcal{L}_{4}=\left[\begin{array}{cc}
\mathbf{Z}_{1} & 0 \\
-\mathbf{Z}_{2}^{\mathrm{T}} & I \\
\mathbf{P}_{11} & \mathbf{P}_{12} \\
\mathbf{P}_{12}^{\mathrm{T}} & \mathbf{P}_{\mathbf{2 2}}
\end{array}\right]\left[\begin{array}{c}
B_{G 2 w} \\
B_{z} D_{G 2 w}
\end{array}\right] \\
& \mathcal{L}_{5}=\left[\begin{array}{ccc}
0 & 0 & 0 \\
0 & 0 & B_{f} \\
I & 0 & 0 \\
0 & I & 0
\end{array}\right] \mathbf{F}\left[\begin{array}{l}
0 \\
0 \\
I
\end{array}\right] \\
& {[\diamond]^{T}\left[\begin{array}{cc|c}
0 & \mathbf{P}_{\mathbf{K}} & 0 \\
\mathbf{P}_{\mathbf{K}} & 0 & 0 \\
\hline 0 & 0 & \mathbf{\Phi}_{\mathbf{1 1}}
\end{array}\right]\left[\begin{array}{cc}
I & 0 \\
A_{K} & B_{K} \\
\hline C_{K} & D_{K}
\end{array}\right]<0} \\
& \operatorname{Trace}(\mathbf{W})<\gamma^{2} \\
& D_{K} D_{q w}=0 \\
& D_{e z} D_{z w}+D_{e f}\left(\left[\begin{array}{ccc}
0 & 0 & I
\end{array}\right] \mathbf{F}[\diamond]^{T}\right) D_{y w}=0
\end{aligned}
$$

with the following matrices:

$$
\left[\begin{array}{c}
A_{G} \\
\hline C_{\Phi} \\
\hline C_{G 2} \\
\hline C_{G 3}
\end{array}\right]=\left[\begin{array}{ccc}
A_{K} & 0 & 0 \\
0 & A_{K} & B_{K} C \\
0 & 0 & A \\
\hline 0 & C_{K} & D_{K} C_{q} \\
C_{K} & 0 & 0 \\
\hline 0 & 0 & C_{z} \\
\hline 0 & 0 & C_{y}
\end{array}\right]
$$




$\left[\begin{array}{c|c}B_{G p} & B_{G w} \\ \hline D_{\Phi p} & D_{\Phi} \\ \hline D_{G 2 p} & D_{G 2 w} \\ \hline D_{G 3 p} & D_{G 3 w}\end{array}\right]=\left[\begin{array}{c|c}B_{K} & 0 \\ B_{K} D_{q p} & B_{K} D_{q w} \\ B_{p} & B_{w} \\ \hline D_{K} D_{q p} & D_{K} D_{q w} \\ D_{K} & 0 \\ \hline D_{z p} & D_{z w} \\ \hline D_{y p} & D_{y w}\end{array}\right]$

Proof: The proof of this theorem can be made using the theorem 2.3 as a starting statement and then the proof of theorem 3.1 can be followed. Make the congruent multiplication as defined in the proof of theorem 3.1, then use the same bijective variable change to get the conditions of the theorem.

Both theorems present conditions to test the existence of a filter completing the plant so that an upper bound on a given worst-case performance is guaranteed. The conditions presented are LMI conditions. For a given $\gamma$ these conditions define a feasibility problem. This can be tested using an efficient algorithm. Furthermore, these constraints can be recast as a minimization problem of a cost function. In this case, minimizing $x=\gamma^{2}$ allows to find the lower upper bound reachable on the worst-case performance with this framework.

If the conditions are feasible, the state space representation of the filter can be reconstructed from the variable change presented in the proof of theorem 3.1, using Packard completion lemma [10] to construct the required matrices.

Cases can be derived from this case. First of all, we recover the case of the robust weighted filtering [15], [14], where the output system $H=\left[\begin{array}{ll}W(j \omega) & -W(j \omega)\end{array}\right]$. In both papers, a variable change is made as $\hat{F}(j \omega)=W(j \omega) F(j \omega)$. The order of the filter synthesized is in this case $n_{F}=2 n_{K}+$ $n_{G}+2 n_{W}$, where $n_{K}$ is the order of the IQC basis, $n_{G}$ the order of the system considered, $n_{W}$ the order of the weightings. The solution presented here allows to have a filter of order $n_{F}=2 n_{K}+n_{G}+n_{W}$, which is cheaper in terms of real-time implementation.

In the case of the robust feed-forward problem, the uncertainty affects the output system $H$ (see figure 1). As a dual problem, this can be solved using the solution presented here. The steps to compute this solution can be found in [15], [7]. The solution proposed for the robust feed-forward problem presents the same advantages as the one for the robust filtering problem. It will be possible to have a reducedorder feed-forward controller and to take into account any transfer function for the input system.

Note that the general case for the robust open-loop problems can be solved using the solution presented in [12]. In this case, it is possible to take into account uncertainties on both the input system $G$, and the output system $H$.

\section{CONCLUSION}

Solutions for the synthesis of a filter for a class of robust open-loop problems have been presented. The filter synthesized ensures an upper bound on the worst-case $\mathrm{H}_{2}$ norm (theorem 3.2), or on the worst case $L_{2}$-gain (theorem 3.1) of the complete open-loop. The uncertainty is modeled using a
LFT representation and taken into account using Dynamic IQCs which allows to consider structured uncertainties of many classes. The synthesis is made through convex optimization problems involving Linear Matrix Inequalities which can be solved efficiently. The results presented here give improved conditions to tackle the robust weighted filtering problem. In addition, it allows to take into account many cases of open-loop plant design. This approach paves the way to a generalized approach of robust open-loop synthesis problems.

\section{REFERENCES}

[1] B. Bayon, E. Blanco, and G. Scorletti. Robust $L_{2}$-Gain Observation for structured uncertainties : an LMI approach. In Proceedings of the 50th IEEE Conference on Decision and Control, Orlando, 2011.

[2] S. Boyd, L. El Ghaoui, E. Feron, and V. Balakrishnan. Linear matrix inequalities in system and control theory. Society for Industrial Applied Mathematics, Philadelphia, 1994.

[3] J.C. Doyle, K. Glover, P.P. Khargonekar, and B.A. Francis. Statespace solutions to standard Hdeux and Hinfini control problems. IEEE Transactions on Automatic control, 34(8):831-847, 1989.

[4] P. Gahinet and P. Apkarian. A linear matrix inequality approach to $H_{\infty}$ control. International Journal of Robust and Non-Linear Control, 4(4):421-448, 1994.

[5] M.P. Kazmerkovski, A. Moradewicz, J. Duarte, E. Lomonowa, and C. Sonntag. Contactless Energy Transfer. In Wilamowski and Irwin, editors, Power Electronics and Motor Drives, chapter 35. Boca Radon, crc press edition, 2011.

[6] A. Khalate, X. Bombois, R. Babuska, and H. Wijshoff. Optimizationbased feedforward control for a Drop-on-Demand inkjet printhead. In American Control Conference, Baltimore, 2010.

[7] I. Kose and C. Scherer. Robust feedforward control of uncertain systems using dynamic IQCs. In Proceedings of the 46th IEEE Conference on Decision and Control, pages 2181-2186, New Orleans, 2007. IEEE.

[8] A. Megretski and A. Rantzer. System analysis via integral quadratic constraints. IEEE Transactions on Automatic Control, 42(6):819-830, 1997.

[9] K. Ohrn, A. Ahlen, and M. Sternad. A Probabilistic Approach to Multivariable Robust Filtering and Open-loop Control. IEEE Transactions on Automatic Control, 40(3):405-417, 1995.

[10] A. Packard, K. Zhou, P. Pandey, and G. Becker. A collection of robust control problems leading to LMIs. In Proceedings of the 30th IEEE Conference on Decision and Control, volume 205, pages 1245-1250, January 1991

[11] Anders Rantzer. On the Kalman-Yakubovich-Popov lemma. Systems and Control Letters, 28(1):7-10, 1996.

[12] C. Scherer. Robust Controller Synthesis is Convex for Systems without Control Channel Uncertainties. In Paul M.J. Hof, Carsten Scherer, and Peter S.C. Heuberger, editors, Model-Based Control: Bridging Rigorous Theory and Advanced Technology, chapter 1, pages 13-31. Springer-Verlag, New-York, 2009.

[13] C. Scherer, P. Gahinet, and M. Chilali. Multi-objective output-feedback control via LMI optimization. IEEE Transactions on Automatic Control, 42(7):896-911, 1997.

[14] C. Scherer and I. Kose. Robustness with dynamic IQCs: An exact state-space characterization of nominal stability with applications to robust estimation. Automatica, 44(7):1666-1675, July 2008.

[15] G. Scorletti and V. Fromion. Further results on the design of robust $H_{\infty}$ feedforward controllers and filters. In Proceedings of the 45th IEEE Conference on Decision and Control, pages 3560-3565, San Diego, 2006.

[16] K. Sun and A. Packard. Robust $H_{2}$ and $H_{\infty}$ filters for uncertain LFT systems. IEEE Transactions on Automatic Control, 50(5):715-720, 2005.

[17] Joost Veenman, H. Koroglu, and C.W. Scherer. An IQC approach to robust estimation against perturbations of smoothly time-varying parameters. In Decision and Control, 2008. CDC 2008. 47th IEEE Conference on, pages 2533-2538. IEEE, 2008.

[18] K. Zhou, J.C. Doyle, and K. Glover. Robust and Optimal Control. Prentice Hall, Upper Saddle River, 1995. 